\title{
Characterization of carboxy methylcellulose doped with DTAB as new types of biopolymer electrolytes
}

\author{
A S SAMSUDIN and M I N ISA* \\ Advanced Materials Research Group, Renewable Energy Research Interest Group, Department of Physical Sciences, \\ Faculty of Science \& Technology, Universiti Malaysia Terengganu, 21300 Kuala Terengganu, Terengganu, Malaysia
}

MS received 1 October 2011; revised 22 February 2012

\begin{abstract}
The investigation of new solid biopolymer electrolyte (BEs) system based on carboxy methylcellulose (CMC) is creating opportunity for new types of electrochemical devices, which may themselves, in turn, revolutionize many industrial areas. Biodegradable carboxy methylcellulose (CMC) doped with dodecyltrimethyl ammonium bromide (DTAB) as BEs were prepared via solution-casting method. Upon addition of 35 wt. \% of DTAB, highest ionic conductivity of $7.72 \times \mathbf{1 0}^{-4} \mathrm{Scm}^{-1}$ was achieved due to its higher amorphous region compared to other samples prepared. This result had been further proven in FTIR study. Temperature dependence relationship obeys the Arrhenius rule from which the activation energy, $E_{\mathrm{a}}$, for ionic conductivity and activation energy for relaxation process, $E_{\tau}$, were evaluated. The divergent values between $E_{\mathrm{a}}$ for ionic conductivity and relaxation process $E_{\tau}$ shows that the ions hop by jumping over a potential barrier.
\end{abstract}

Keywords. Cellulose; Fourier transform infrared (FTIR) spectroscopy; X-ray diffraction (XRD); electrical properties; relaxation process.

\section{Introduction}

Global warming is a worrying environmental issue, which has resulted in active public movement to reduce environmental loads. The use of 'eco-friendly' materials as conducting polymer electrolyte (PE) can certainly contribute to the sustainability of the planet. Since the early studies of Wright (1975) and Armand et al (1978) on polymer-salt complexes, PE has been extensively studied as an all-solid-state functional device. PE has advantages over liquid electrolyte such as light weight, improve leakage problem, mechanically stable and flexible for packaging design. Ionically conductive PE has become the key object of academic and industrial interests. They also offer numerous advantages, for example, eliminate corrosive solvent and harmful gas formation, wider electrochemical and thermal stability range as well as low volatility with easy handling (Adebahr et al 2003).

PE consists of salts dissolved in solid polymers, and the polymer must contain a Lewis base which serves to coordinate the cations, thus promoting dissolution of the salt (Karan et al 2008). However, the cation-Lewis base bonding must be labile to allow ion mobility (Gray 1991). Thus, several essential characteristics are necessary for a polymer to act as a successful host in PEs system. This criterion (Ratner 1987; Gray 1991) has been laid down as a guide for the selection of polymer materials. Recently, biodegradable materials have attracted enormous attention worldwide as a result of white pollution, one of the environmental hazards. In addition, it could overcome the main shortcoming

\footnotetext{
*Author for correspondence (ikmar_isa@umt.edu.my)
}

of synthetic polymer, which is mostly insoluble in solvents (Ma et al 2007). Therefore, much effort has been made to develop biopolymer electrolytes by using natural polymer. Several renewable resource-based biopolymers are suitable to be used as host polymer in the polymer electrolytes, such as cellulose (Pawlicka et al 2004), starch (Lopes et al 2003), chitosan (Wan et al 2006; Majid and Arof 2007) and natural biodegradable polymers (Singh et al 2010) that have been studied as conducting PE and applied in electrochemical devices such as batteries, supercapacitors, fuel cells, solar cells and electrochromic windows.

Carboxy methylcellulose (CMC) is a cellulose derivative with carboxymethyl groups bound to the hydroxyl groups of the glucose unit (Guo et al 1998). CMC is an industrially important cellulose derivative. Examples of industrial applications are found in the food industry as a thickener and a binder, in the oil industry as a lubricant for drilling, and in the cosmetic industry as a stabilizer and a binder. Because of its non-toxicity, biodegradability and biocompatibility, CMC solution has also been widely used as a carrier for bone graft (Santa-Comba et al 2001) Normally, polymer electrolytes have very poor conductivity due to their dual nature (partially amorphous and partially crystalline) (Pandey et al 2008). To enhance the ionic and mechanical properties of PEs, various attempts have been made in the past decade with the addition of different salts and fillers with the latter leading to formation of composite polymer electrolyte. The polymers have been complexed with various salts, which provide the ions for conduction. Ammonium salts have already been reported as a good proton donor to the polymer matrix (Majid and Arof 2005; Stainer et al 1984; Daniel et al 1988). 
In the present work, dodecytrimethyl ammonium bromide (DTAB) has been chosen as the dopant salt in the development of CMC-salt complexes based biopolymer electrolytes (BEs) system. The effect of the dopant salt composition on the ionic conductivity of the CMC-DTAB BEs at ambient temperature was studied based on the structural and electrical properties via Fourier transform infrared (FTIR) spectroscopy, X-ray diffraction (XRD) and electrical impedance spectroscopy (EIS).

\section{Experimental}

\subsection{Sample preparation}

$2 \mathrm{~g}$ of CMC obtained from Acros Organic Co., was dissolved in distilled water. Then, varied amounts of DTAB in weight \% (5-40 wt. \%) was added. The mixture was stirred continuously until complete dissolution of DTAB became homogenous. The mixture was then poured into different Petri dishes and left dry at room temperature for the film to form. The films were kept in desiccators for about 1 month for ambient drying before being characterized to ensure no water was present in the BEs system. To reduce the drying time, samples can be dried in vacuum oven. The CMC-BEs were cut into suitable sizes for further characterization.

\subsection{FTIR spectroscopy}

FTIR spectroscopy measurement was carried out using Thermo Nicolet 380 FTIR spectrometer. The spectrometer was equipped with an Attenuated Total Reflection (ATR) accessory with a germanium crystal. The sample was placed on a germanium crystal. The samples were analysed in the frequency range from 4000 to $675 \mathrm{~cm}^{-1}$ with spectra resolution of $4 \mathrm{~cm}^{-1}$.

\section{$2.3 X$-ray diffraction}

To study the nature of BEs system, X-ray diffraction (XRD) measurements were performed using Rigaku MiniFlex 2. Prior to analysis, samples were cut into a suitable size $(2 \times 2 \mathrm{~cm})$ and then adhered onto a glass slide. The glass slide was then placed in the sample holder of the diffractometer and the samples were directly scanned at $2 \theta$ angles between $5^{\circ}$ and $80^{\circ}$ with X-rays of $1.5406 \AA$ wavelength generated by a $\mathrm{Cu} \mathrm{K} \alpha$ source.

\subsection{Impedance analysis}

The biopolymer CMC-DTAB electrolyte film was sandwiched between stainless steel and impedance measurements were performed with electrical impedance spectroscopy (HIOKI 3532-50 LCR Hi Tester) in the frequency range $50 \mathrm{~Hz}-1 \mathrm{MHz}$ between ambient temperature and elevated temperature. The effect of DTAB composition on conducti- vity was analysed based on the frequency dependent electrical properties $\left(\varepsilon^{*}, M^{*}\right.$ and $\left.\tan \delta\right)$ using the equation explained in Samsudin and Isa (2012a, b), Buraidah and Arof (2011) and Majid and Arof (2005).

\section{Results and discussion}

FTIR analysis was performed to obtain structural, compositional and bonding information (complexation) concerning BEs system. FTIR spectrum in the range of 700-2500 $\mathrm{cm}^{-1}$ as shown in figure 1(a) represents interaction of CMC with the variation of DTAB concentration. In the present work, the carboxyl band of pure CMC film can be found at $1602 \mathrm{~cm}^{-1}$.

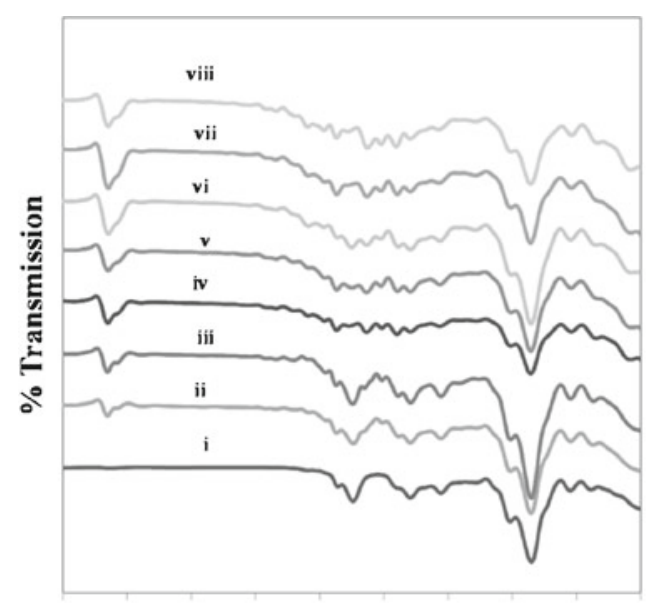

$\begin{array}{llllllllll}2500 & 2300 & 2100 & 1900 & 1700 & 1500 & 1300 & 1100 & 900 & 700\end{array}$

wavenumber $\left(\mathrm{cm}^{-1}\right)(\mathrm{a})$

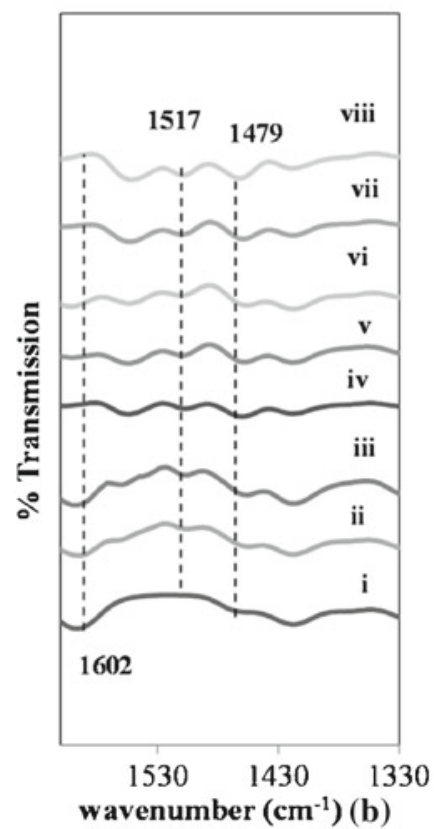

Figure 1. FTIR spectrum of (i) CMC film and CMC doped with (ii) 5 wt. $\%$, (iii) 10 wt. $\%$, (iv) 15 wt. $\%$, (v) 20 wt. $\%$, (vi) 25 wt. $\%$, (vii) 30 wt. $\%$ and (viii) 35 wt. $\%$ of DTAB. 
In previous reports (Abdel et al 1994; Pushpamalar et al 2006), the band at $1602 \mathrm{~cm}^{-1}$ is assigned to asymmetrical $\mathrm{COO}^{-}$stretching of the carboxylate anion in the CMC. With the addition of DTAB in the system, this peak is expected to be effected due to the lone pair of electrons that attract the salt molecules to it (Ali et al 1998). The possible interaction between CMC and doping DTAB is highlighted in figure 1(b).

From figure 1(b), it can be observed that upon addition of DTAB in the CMC-BEs system, the carboxyl group at $1602 \mathrm{~cm}^{-1}$ has shifted to a lower wavenumber of $1564 \mathrm{~cm}^{-1}$. The shifted peak of $38 \mathrm{~cm}^{-1}$ in magnitude is believed to be due to $\mathrm{COO}^{-}$of the CMC. A new peak is observed at $1517 \mathrm{~cm}^{-1}$ which indicates the presence of bending vibration of $\left(\mathrm{CH}_{2}\right)$ alkyl group in DTAB. The intensity of this peak increased with increment in DTAB concentrations. In addition, the peak at $1479 \mathrm{~cm}^{-1}$ has shifted to the lower wavenumber at $1459 \mathrm{~cm}^{-1}$ with an increase of DTAB concentration which indicated that interaction occurred between $\mathrm{CMC}$ and DTAB. This band is the assignment of $\mathrm{CH}_{3}$ bending vibration in DTAB (Wang et al 2008). From this observation, there is a strong contribution of hydrogen bonding and coordination interaction of $\mathrm{H}^{+}$to the $\mathrm{COO}^{-}$between CMC and DTAB. Hashmi et al (1990) and Hema et al (2008) have proven that in polymer-ammonium salt system, the conducting species is $\mathrm{H}^{+}$ion which originates from the ammonium ion. This study again proves that in a similar system, $\mathrm{H}^{+}$ion was found to be a conducting species. The conduction is believed to occur through structure diffusion (Grotthus mechanism) where the exchange of ions take place between the complexed sites (Samsudin and Isa 2012a, b). Proton conduction by the Grotthus mechanism involves intermolecular proton jump creating a vacant site followed by reorientation to occupy the vacant site. In this present case, lone proton migration $\left(\mathrm{H}^{+}\right)$mechanism is more probable because of the following explanation. Two of the four $\mathrm{NH}_{4}^{+}$hydrogen ions are bound identically, one hydrogen is bound more strictly and the fourth more weakly. The weakly bound $\mathrm{H}^{+}$ of $\mathrm{NH}_{4}^{+}$can easily be dissociated. These $\mathrm{H}^{+}$ions can hop via one site to another leaving a vacancy which will be filled by another $\mathrm{H}^{+}$ion from a neighbouring site (Hashmi et al 1990; Buraidah et al 2009; Kadir et al 2011). These entire events occurred in the coordinating site (oxygen) of the host polymer (CMC) and thus conduction took place (Hema et al 2008). Hence, from the FTIR spectroscopic analysis, interaction between CMC and DTAB can be confirmed and the conduction mechanism in BEs is expected to occur via the Grotthus mechanism which suggests that the charge carrier in this system is predominantly due to ion which is $\mathrm{H}^{+}$as proven from the previous work studied on the conduction mechanism of the same system (Samsudin and Isa 2012b).

Figure 2 shows XRD patterns of BEs based on CMCDTAB at different compositions respectively. XRD pattern of DTAB (figure 2(i)) shows intense peak at $2 \theta=8.85^{\circ}$, $17.07^{\circ}, 21.06^{\circ}, 25.36^{\circ}, 29.56^{\circ}$ and $38.14^{\circ}$, which reveals the crystalline nature of DTAB. The broad peak at $20 \cdot 25^{\circ}$ (figure 2(x)) confirms the amorphous nature of pure CMC

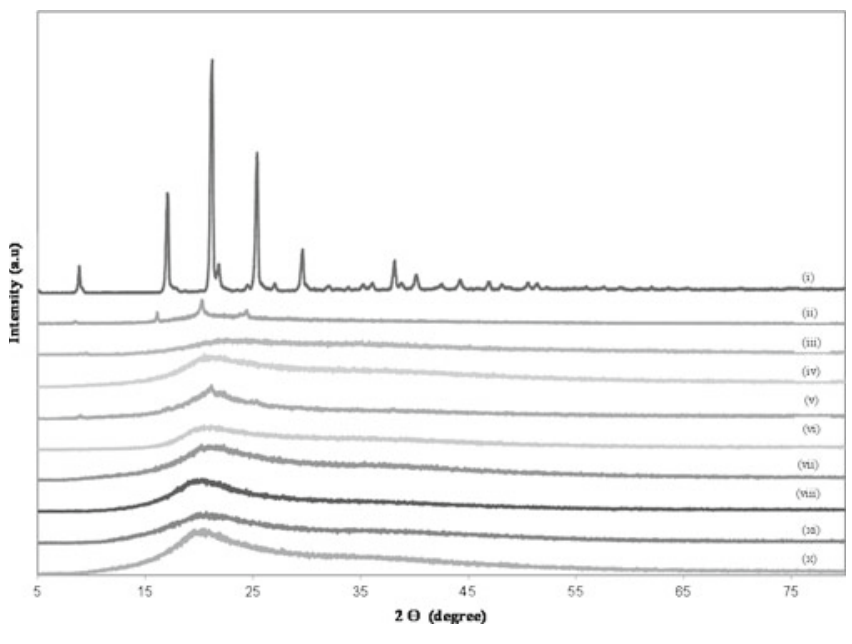

Figure 2. XRD patterns for (i) pure DTAB, (ii) CMC-40 wt. \% DTAB, (iii) CMC-35 wt. \% DTAB, (iv) CMC-30 wt. \% DTAB, (v) CMC-25 wt. \% DTAB, (vi) CMC-20 wt. \% DTAB, (vii) CMC15 wt. \% DTAB, (viii) CMC-10 wt. \% DTAB, (ix) CMC-5 wt. \% DTAB and (x) CMC pure films.

film. This broad peak is known as the 'amorphous hump' and is a typical characteristic of amorphous materials. The relative intensity of broad peak between $11^{\circ}$ and $27.21^{\circ}$ decreases with the increase in salt composition and this may be due to the interaction of the salt with the polymer, resulting in increasing of amorphousness of the BEs system. It can be seen from the X-ray diffractograms that the amorphousness of the polymer electrolyte decreases with the addition of more than 35 wt. \% of DTAB. Presence of these peaks at $2 \theta=16 \cdot 16^{\circ}, 20 \cdot 30^{\circ}$ and $24.74^{\circ}$ implies that the polymer could no longer solvate the salt because of which crystallinity of BEs is expected to increase. Changes in amorphousness of the CMC-BEs system affected conductivity of the samples. This amorphous nature was found to be responsible for the greater ionic diffusivity resulting in high ionic conductivity (Balasubramanyam et al 2007; Shuhaimi et al 2010). This observation confirms that more complexation has taken place in amorphous phase compared to less amorphous phase (sample $>35$ wt. \% of DTAB).

The impedance spectra (Cole-Cole plot) of BEs system with difference concentration of DTAB is depicted in figure 3. For samples containing 0 and $10 \mathrm{wt}$. \% DTAB (figures 3(a) and (b)) two well defined regions can be observed, a high frequency incomplete semicircle followed by a spur (an inclined spike) at the low frequency region. The semicircle observed in the figures are tilted, indicating that the ions have plural relaxation time. The semicircle is related to the relaxation process in the bulk polymer electrolyte (Woo et al 2011) and the appearance of semicircle can be explained by a parallel combination of resistor and capacitor. In the present work, the resistor is referred to the migration of the ions through the polymer matrix and the capacitor representing the polarized immobile polymer chains in the alternating field (Ramya et al 2006) which is circuit network where the capacitance is usually of the order 

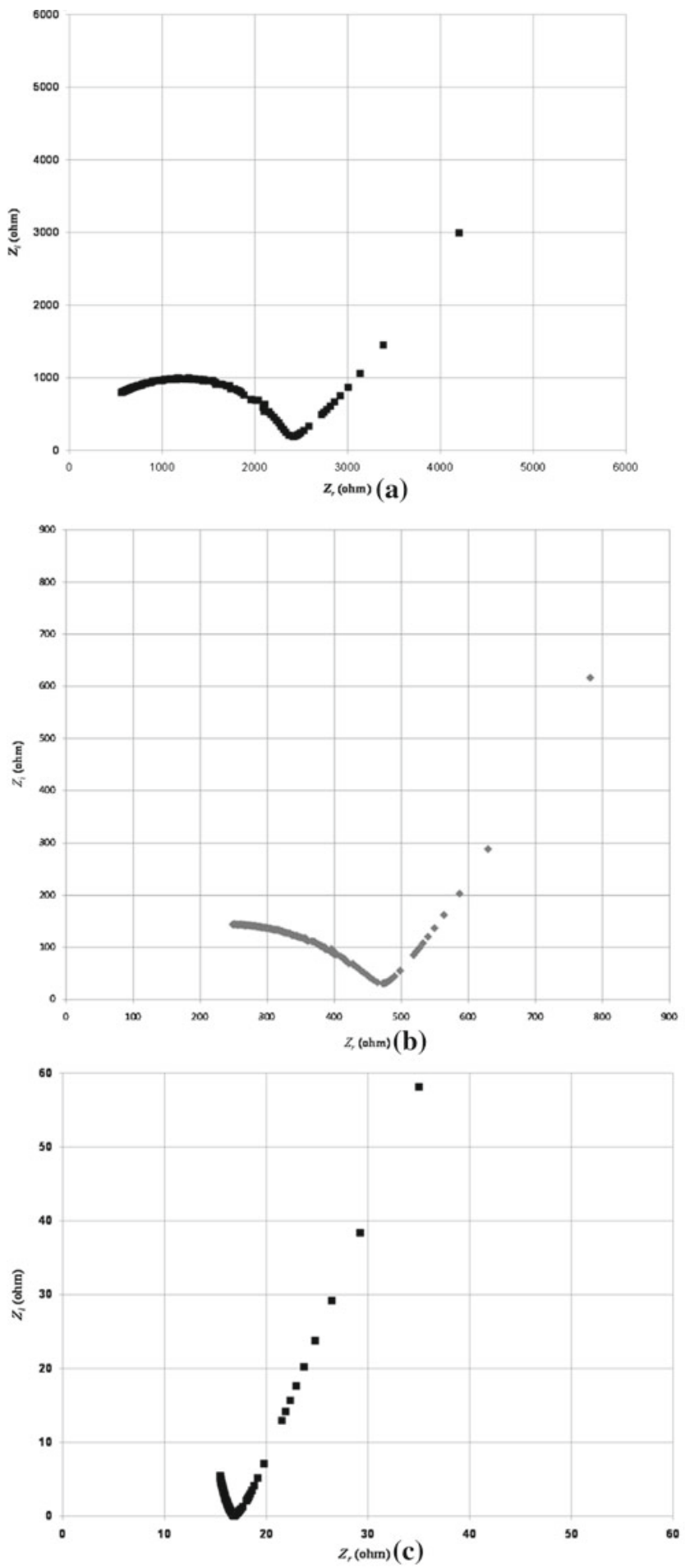

Figure 3. Cole-Cole plot for (a) CMC-0 wt. \%, (b) CMC10 wt. $\%$ and (c) CMC-35 wt. \% of DTAB at ambient temperature.

of nano-Farads (Nadeem et al 2002). The spur at low frequency of the spectra is attributed to the effect of electrode polarization (Hema et al 2008). The inclination of the spur parallel to the imaginary axis is due to the double layer capacitance at the blocking electrodes (Kim et al 1999) and is better known as the polarization effect at electrode/ electrolyte interface (Song et al 2000). It is noted that the semicircle observed at high frequencies completely disappears in the BEs with increment of DTAB, as shown in figure 3(c). This suggests that only the resistive component of the polymer prevails (Hema et al 2008). With increasing salt composition, a local effective pathway is constructed in the liquid phase for ionic conduction, as a result, ions can transport quickly in the liquid phase as the electric potential alternates between the positive and negative electrodes in an a.c. field. The bulk resistance $\left(R_{\mathrm{b}}\right)$ was retrieved from the intercept of the straight line on the $Z^{\prime}$ axis and the ionic conductivity, $\sigma$, of the sample can be calculated from the equation:

$$
\sigma=\frac{t}{R_{\mathrm{b}} A} \text {. }
$$

Here $t$ and $A$ are thickness and area of the samples, respectively and $R_{\mathrm{b}}$ the bulk resistance determined from EIS spectra. The values of bulk resistance and conductivity, $\sigma$, are tabulated in table 1 . From table 1 , it can be observed that with the increase of DTAB composition, the $R_{\mathrm{b}}$ value decreases and hence the conductivity increases. The decrease in resistance of polymer electrolyte is due to the increment in the number of charge carriers of free ions (which is $\mathrm{H}^{+}$as shown previously) and its ionic mobility (Ramly et al 2011; Samsudin et al 2011). From XRD results, increase in ionic conductivity with increasing salt concentration can be attributed to the increase in the amorphousness of the sample. It is inferred that the sample containing $35 \mathrm{wt}$ \% DTAB is the most amorphous sample, thus exhibiting the highest ionic conductivity. In contrast, decrease in conductivity with the increment of salt content $>35$ wt. \% could be attributed to the formation of ion pairs and aggregates. The formation of ion also produces neutral species and thus reduces the number of free ions (Schantz and Torell 1993; Teeters et al 1996) and also the excess salt recrystallized out of the polymer as proven by XRD result as shown in figure 2(ii).

Figure 4 shows the $\log \sigma-1000 / T$ plot for various compositions of CMC-DTAB based BEs in the temperature range 303-393 K. The plot shows that the ionic conductivity of BEs system increases with increasing temperature for all salt compositions which are thermally assisted (Majid et al 2005; Idris et al 2009; Samsudin and Isa 2012a, b). The $\log \sigma$ vs $1000 / T$ plot for the CMC-BEs system shown in figure 4 confirms that the ionic conductivity of the biopolymer electrolyte increases with increasing temperature for all compositions. The regression values are close to unity $\left(R^{2} \sim 1\right)$, suggesting that the temperature-dependent ionic conductivity for all complexes obeys Arrhenius behaviour (Rajendran and Uma 2000; Samsudin et al 2011). Since all polymer complexes do not show any abrupt jump with temperature, it indicates that these electrolytes exhibit a completely amorphous structure (Michael et al 1997). In general, it is believed that the conductivity increases as the degree of crystallinity decreases (Shuhaimi et al 2010) or, in other words, the flexibility of the polymeric backbone increases. The crystalline regions obstruct ion movement by blocking the paths to ions. The change of conductivity with 
Table 1. Calculated value of $\sigma, E_{\mathrm{a}}$ and $E_{\tau}$ for CMC-DTAB systems.

\begin{tabular}{|c|c|c|c|c|}
\hline Sample CMC + DTAB (wt. \%) & $R_{\mathrm{b}}(\Omega)$ & $\sigma\left(\mathrm{Scm}^{-1}\right)\left(\times 10^{-5}\right)$ & $E_{\mathrm{a}}(\mathrm{eV})$ & $E_{\tau}(\mathrm{eV})$ \\
\hline $\mathrm{CMC}+$ DTAB (0 wt. \%) & $2410 \cdot 00$ & $0 \cdot 38$ & $0 \cdot 20$ & 0.45 \\
\hline $\mathrm{CMC}+$ DTAB (5 wt. $\%)$ & $967 \cdot 00$ & 1.03 & $0 \cdot 18$ & $0 \cdot 38$ \\
\hline CMC + DTAB (10 wt. \%) & $457 \cdot 00$ & $2 \cdot 09$ & $0 \cdot 17$ & $0 \cdot 34$ \\
\hline CMC + DTAB (15 wt. \%) & $146 \cdot 00$ & $6 \cdot 54$ & $0 \cdot 14$ & $0 \cdot 31$ \\
\hline CMC + DTAB (20 wt. \%) & $90 \cdot 40$ & $10 \cdot 90$ & $0 \cdot 13$ & $0 \cdot 25$ \\
\hline CMC + DTAB (25 wt. \%) & $36 \cdot 10$ & $30 \cdot 00$ & $0 \cdot 11$ & $0 \cdot 20$ \\
\hline CMC + DTAB (30 wt. \%) & $23 \cdot 60$ & $51 \cdot 30$ & $0 \cdot 10$ & $0 \cdot 19$ \\
\hline CMC + DTAB (35 wt. \%) & $16 \cdot 50$ & $77 \cdot 20$ & 0.09 & $0 \cdot 17$ \\
\hline CMC + DTAB (40 wt. \%) & $82 \cdot 80$ & $14 \cdot 60$ & $0 \cdot 13$ & $0 \cdot 27$ \\
\hline
\end{tabular}

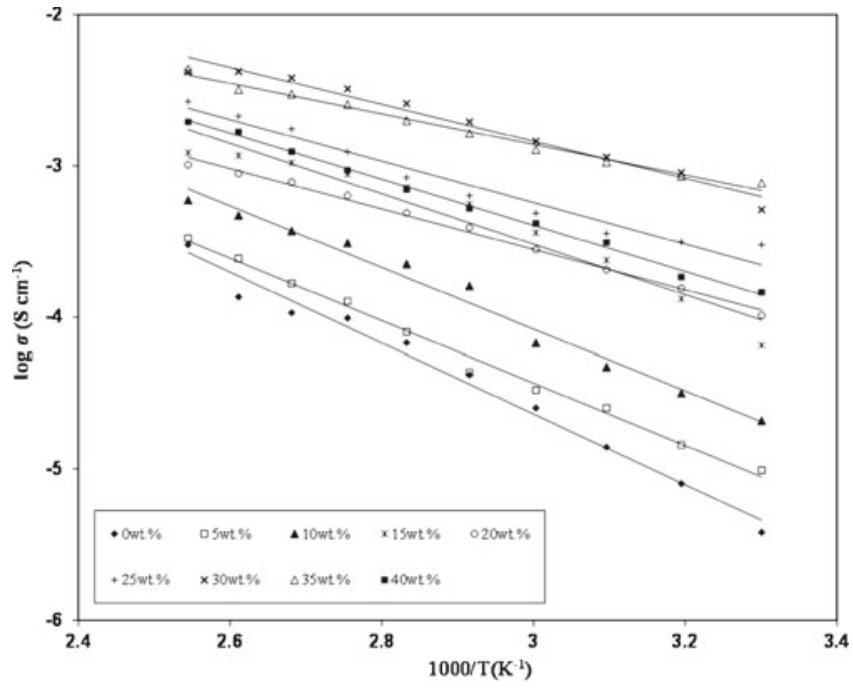

Figure 4. Plot of $\log \sigma$ vs $10^{3} / T$ for CMC-DTAB BEs system.

temperature is explained in terms of polymer segmental motion, which results in an increase of free volume (Miyamoto and Shibayama 1973; Druger et al 1983). This can be understood with the emphasis that as temperature increases, the vibrational energy of segmental motion operates against the hydrostatic pressure imposed by its neighbouring atoms. Consequently, it creates a small amount of space surrounding its own volume in which vibrational motion can occur. Therefore, free volume around the polymer chain causes amplification in charge carrier and mobility of ions and hence enhances the conductivity. This, in turn, favours the hopping of inter-chain and intra-chain ion movements and conductivity of the polymer electrolyte thus becomes high (Hashmi et al 1992; Sreepathi Rao et al 1995; Jaipal Reddy and Chu 2002). This can also be linked to decrease in viscosity and, hence, increase in chain flexibility (Sekhon et al 1998). As temperature increases, the nature of cation transport is quite similar to that in ionic crystals, where ions jump into neighbouring vacant sites and hence, increase the ionic conductivity to higher value (Souquet et al 1994). Similar results have also been reported for different types of polymer electrolytes (Hashmi et al 1990; Maurya et al 1992).
Since the plot was considered as Arrhenius behaviour, it can be represented by the relation,

$$
\sigma=\sigma_{0} \exp \left(\frac{-E_{\mathrm{a}}}{k T}\right)
$$

where $\sigma_{0}$ is the pre-exponential factor, $E_{\mathrm{a}}$ the activation energy and $k$ the Boltzmann constant.

The activation energy, $E_{\mathrm{a}}$, is listed in table 1 which is calculated by linear fit of the conductivity data shown in figure 4. $E_{\mathrm{a}}$ is the energy required to move the ions, presupposing that the structure remains unchanged, plus the energy required to deform the structure enough to allow the ion to pass. Based on the $E_{\text {a }}$ values, it can be deduced that the conduction process decreases gradually with increasing DTAB composition which implies that the ions in highly conducting samples require lower energy for migration. Since the ion transfer is greatly affected by the polymer segmental motion (Ramesh and Arof 2000); an electrolyte with lower value of $E_{\mathrm{a}}$ implies rapid ionic conduction and then increases conductivity to the higher value. The low activation energy for CMC-DTAB BEs system ion transport is due to the completely amorphous nature of polymer electrolyte that facilitates the fast $\mathrm{H}^{+}$ion motions in the polymer network as supported by XRD results.

DTAB composition dependence of the dielectric constant $\left(\varepsilon_{\mathrm{r}}\right)$ at selected frequencies is shown in figure 5(a). From the plot (figure 5(a)), $\varepsilon_{\mathrm{r}}$ increases as the increment of DTAB composition for every frequency until $35 \mathrm{wt}$. \%. This implies that, as the DTAB composition increases, the store charge is increased which means that the number of charge carriers has increased causing asymptotic rise in dielectric constant values. In all of the salt composition variants, a strong frequency of $\varepsilon_{\mathrm{r}}$ was observed in the low frequency followed by nearly frequency independent behaviour with increasing frequency. The decrease of $\varepsilon_{\mathrm{r}}$ with increase in frequency may be attributed to the electrical relaxation process (Pradhan et al 2009). As the frequency increases, the ionic and orientation source of polarizability decrease and finally disappears due to inertia of mobile ions, leading to the observed decrease in $\varepsilon_{\mathrm{r}}$. Figure 5(b) shows variation of dielectric loss $\left(\varepsilon_{\mathrm{i}}\right)$ at selected frequencies for different salt compositions. $\varepsilon_{\mathrm{i}}$ is also seen to decrease with increasing frequency at room temperature. The increase of $\varepsilon_{\mathrm{i}}$ at low 

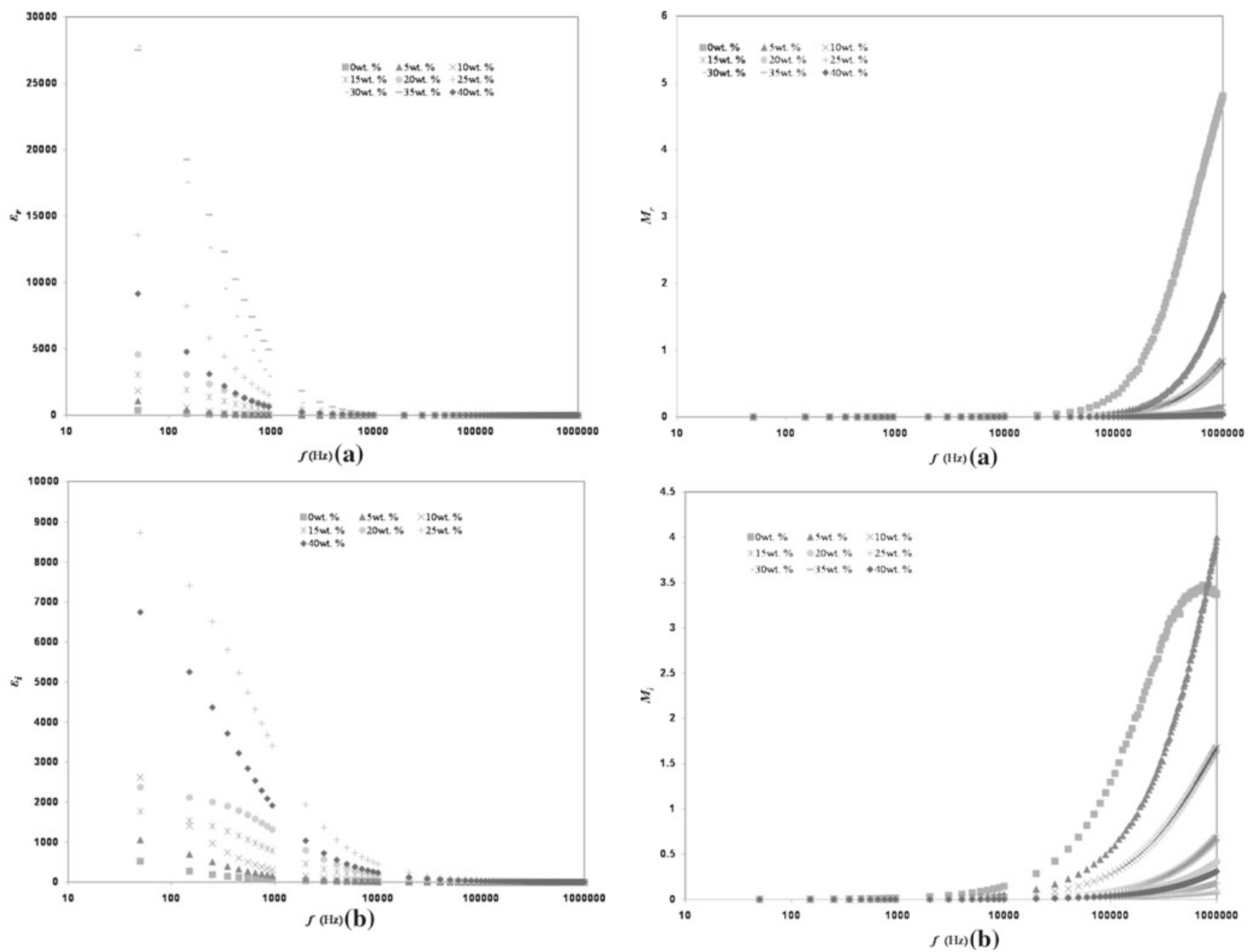

Figure 5. Dipersion of dielectric for CMC-DTAB BEs system: (a) dielectric constant vs frequency and (b) dielectric loss vs frequency.

frequency is due to the free charge motion within the electrolytes (Pandey et al 2010). On enhancement in salt composition, higher value of $\varepsilon_{\mathrm{i}}$ reflects enhancement in mobility of charge carrier. In the high frequency, direction of the electric field changes at a faster rate. Hence, most of the ions will be in the bulk of each sample and lesser ions can pile up at the interface. Therefore, the value of $\varepsilon_{\mathrm{i}}$ decreases with increase in frequency.

The real $\left(M_{\mathrm{r}}\right)$ and imaginary $\left(M_{\mathrm{i}}\right)$ parts of modulus formalism measured as functions of frequency for CMC-DTAB BEs system complexes at ambient temperature are shown in figure 6. From the plots, it shows that the real $\left(M_{\mathrm{r}}\right)$ and imaginary $\left(M_{\mathrm{i}}\right)$ increased at the high frequency end due to the electrode polarization phenomena. With the increment of salt composition, $M^{*}$ shifted to a lower value with increasing frequency which is due to delocalized long-range conductivity and suggesting a plurality of relaxation mechanism for BEs system (Mishra et al 1998). The possible presence of peaks in the modulus formalism at higher frequencies for all the BEs system indicates that it is an ionic conductor

Figure 6. Dipersion of electrical modulus for CMC-DTAB BEs systems: (a) real part of electrical modulus vs frequency and (b) imaginary part of electrical modulus vs frequency.

(Ramesh and Arof 2001). The peaking curve at higher frequencies (figure 6(b)) may be attributed to the bulk effect. At low frequency, $M_{\mathrm{r}}$ and $M_{\mathrm{i}}$ approach zero indicating a large association between the values of capacitance with the electrodes (Muralidharan et al 2005). The plots also give a long tail at lower frequencies which further confirms the nonDebye behaviour in CMC-DTAB BEs (Gogulamurali et al 1992; Razak and Winnie 2009). However, no definitive peaks can be observed from the plot as shown in figure 6. Thus, $\tan \delta$ formalism was plotted with respect to frequency for CMC-DTAB BEs system.

Figure 7 depicts frequency dependence of loss tangent (tan $\delta$ ) for CMC-DTAB BEs system at ambient tempereature. It can be seen that as salt content increases, the maximum of $\tan \delta$, $(\tan \delta)_{\max }$, shifts towards higher frequencies. Meanwhile, the maximum height also increases with additional salt composition, suggesting the presence of dielectric relaxation process in the material. This type of relaxation is called dipolar group relaxation (Pandey et al 2010). The occurrence of relaxation process is the result of the efforts 


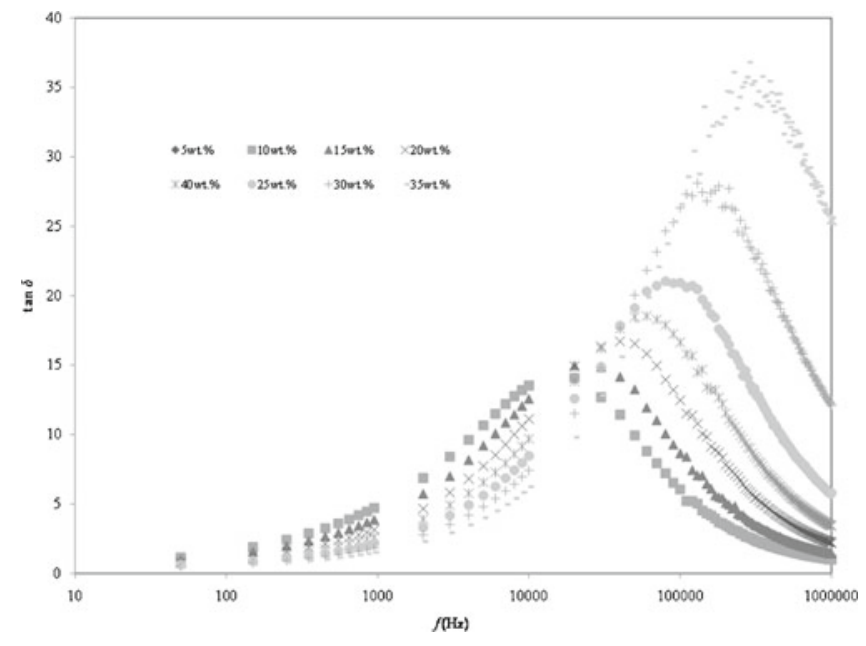

Figure 7. Loss tangent vs frequency for CMC-DTAB BEs systems.

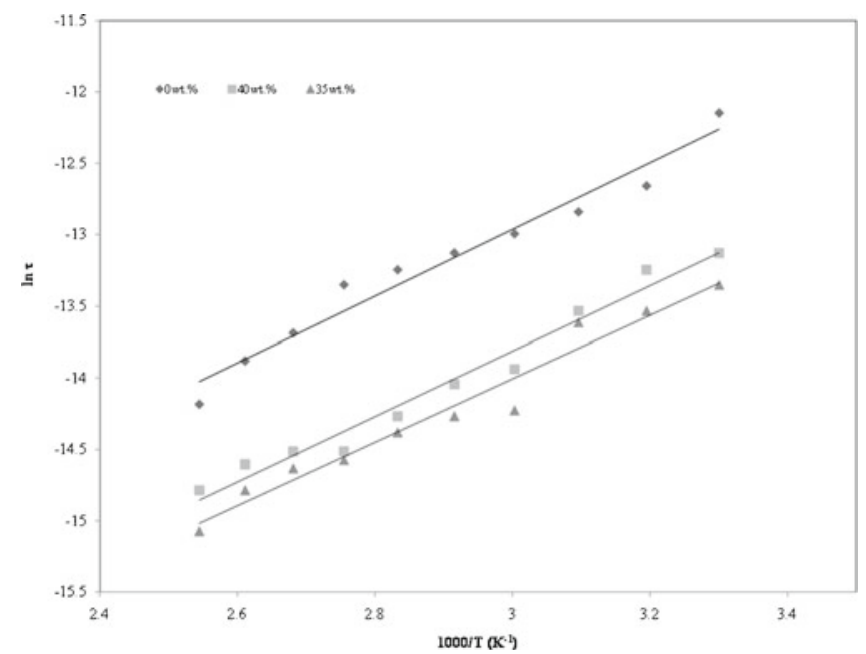

Figure 8. Variation of relaxation time, $\tau$, with reciprocal temperature for $\mathrm{CMC}-\mathrm{DTAB} \mathrm{BEs}$ systems.

carried out by ionic charge carriers within the polymer material to obey the change in the direction of the applied field. With the addition of salt composition in polymer matrix, it enhances the amorphousity of the material where molecular packing is loose and weak. Thus, the chain in this phase is more flexible and is capable of orienting themselves relatively easily and rapidly. The angular frequency of the applied field, $\omega_{\max }$, at which the $f_{\max }$ occurs is from $\tan \delta$ peak frequencies of figure 7 , defining the relaxation time for the ionic charge carriers, $\tau$, by the relation:

$$
\tau \omega_{\max }=1 .
$$

The relaxation time, $\tau$, for various compositions of salt is shown in figure 8 as an Arrhenius function of temperature. The occurrence of relaxation time results from the efforts carried out by ionic charge carriers within the polymer material obeying the change in the direction of applied field. As the temperature increases, the ions acquire more kinetic energy and thus experience an increase in mobility. The ions with increased mobility will be able to align themselves with the applied field in a shorter time when the field changes direction. Thus, relaxation time of the ions decreases with temperature which is assisted by the increasing degree of salts dissociation and enhances the charge carrier as well (El Shafee 1996; Ramly et al 2011). The decrease of $\tau$ with increasing temperature also contributed to the decrease of resistivity of the samples due to the increment in the number of charge carriers of conduction (Prabakar et al 2003).

The effect of DTAB composition on $\tau$ can be explained by the fast and slow relaxation processes from the peaks whose temperature dependence is noted. With the addition of DTAB compositions, it can be found that it is affecting $\tau$ values where the fast relaxation process rapidly occurs in CMCDTAB BEs system which are unperturbed by the solvated salt (in fast relaxation process, $\mathrm{H}^{+}$ions have the strength to release). With the addition of salt, it provides higher numbers of cation which is responsible for fast segmental motion (Bergman et al 1995) of the polymeric chain thus reducing the relaxation time and increasing the transport properties and as a result, sample with $35 \mathrm{wt}$. \% of DTAB exhibits the highest electrical conductivity. The slow process is observed for large salt composition and with addition of salt composition more than $35 \mathrm{wt}$. \% shows that the ionic conductivity decreased. In this situation, crosslink chain segment due to the $\mathrm{H}^{+}$ions slow down the segmental mobility which supports assignment of the slow process to salt rich region where there are no unperturbed "free" segmental motions (Bergman et al 1995).

The variation of relaxation time, $\tau$, with temperature can be described by an Arrhenius plot and is given as

$$
\tau=\tau_{0} \exp \left(E_{\tau} / k T\right)
$$

where $E_{\tau}$ is the activation energy for the relaxation process, $k$ the Boltzmann constant and $\tau_{0}$ the pre-exponential factor. The shift of $(\tan \delta)_{\max }$ towards higher frequency with increasing conductivity indicates that activation energy for the relaxation process decreases with conductivity as shown in table 1.

From table 1 it can be observed that $E_{\tau}$ is influenced by salt composition where the value decreases with the increase in salt composition. The relaxation of charge carriers can be associated with the migration of carriers in the sample which have to overcome less energy barrier during relaxation process compared to conduction process. The decreased values of $E_{\tau}$ confirmed that the hopping conduction mechanism is the predominant mechanism in these samples (Abo et al 2003). Due to the increasing composition of DTAB resulted in increase of the density of states (i.e. the concentration of polarons) and according to Migahed et al (2004), the polaron concentration favours the probable transition between the 
states. The decrease in $E_{\tau}$ can be accounted for by polaron hopping theories. According to Ahmad et al (2003), if the activation energy for the d.c. conductivity and relaxation process is about the same (small difference); a conductivity contributing ion has to overcome the same barrier while conducting as well as relaxing. The small difference may be due to the fact that the relaxation process involved only the hopping energy of the carriers between localized states. But, in this work, activation energy of the relaxation is double the activation energy of conduction. This can be inferred that the conducting ions in the CMC-DTAB BEs system need more relaxing process before the conduction processes occur. Since the conductivity-temperature relationship for the CMC-DTAB BEs system is Arrhenian, the ionic conduction is predicted through hopping mechanism (Agrawal and Pandey 2008) (where the displacement of an ion from one site to another neighbour site) and this hopping mechanism besides jumping over a potential barrier also can be accompanied by some model of hopping mechanism (Psarras 2006).

\section{Conclusions}

The investigation of new biopolymer electrolytes based on CMC-DTAB has been prepared by solution cast technique that takes about $\sim 1$ month to form. In order to minimize the drying process, vacuum oven can be used which will reduce the drying process. FTIR spectroscopy provides an insight into the possible interaction between CMC and DTAB which confirm via Grotthus mechanism and the ionic species in the polymer electrolytes has been well established as a proton conductor $\left(\mathrm{H}^{+}\right)$. The composition of DTAB has been found to influence the ionic conduction of the BEs system. The highest room temperature ionic conductivity for the system is $7.72 \times 10^{-4} \mathrm{Scm}^{-1}$ for sample containing $35 \mathrm{wt}$. $\%$ of DTAB. The decrease in conductivity at higher DTAB composition (>35 wt. \%) can be attributed to the lower number of density of mobile ions in the bulk electrolytes. Due to high DTAB concentration in these electrolytes, the ions have resurfaced and crystallized on the film surface which was supported by the X-ray diffraction patterns. From the XRD patterns, it clearly indicates peaks due to the presence of DTAB crystal in the BEs system. Temperature dependence of ionic conductivity and relaxation time of these systems exhibited Arrhenius behaviour. The activation energy values obtained from ionic conductivity and relaxation time plots against reciprocal temperature for the CMC-DTAB based BEs system shows that the ions hop by jumping over a potential barrier.

\section{Acknowledgements}

One of the authors (ASS) would like to thank UMT Graduate School for BTC scholarship, MOHE for the FRGS Vot59185, Department of Physical Sciences and Universiti Malaysia Terengganu for the technical and research support.

\section{References}

Abdel H, Afkar K, Hosny, Wafaa Basta M, Altaf H and El-Saied H 1994 Polym.-Plast. Technol. 33781

Abo A M, El Ata, Attia S L and Meaz T M 2003 Solid State Sci. 6 61

Adebahr J, Byrne N, Forsyth M, MacFarlane D R and Jacobsson P 2003 Electrochim. Acta 482099

Agrawal R C and Pandey G P 2008 J. Phys. D Appl. Phys. 41514

Ahmad M M, Yamada K and Okuda T 2003 Physica $B 33994$

Ali A M M, Mohamed N S and Arof A K 1998 J. Power Sources 74 135

Armand M B, Chabagno J M and Duclot M 1978 Extended abstract: Second international meeting on solid electrolytes, St Andrews, Scotland (Amsterdam: North Holland Publishers)

Balasubramanyam A V, Reddy T J R, Sharma A K and Narasimha Rao V V R 2007 Ionics 13349

Bergman R, Brodin A, Engberg D, Lu Q, Angell C A and Torell L M 1995 Electrochim. Acta 402049

Buraidah M H and Arof A K 2011 J. Non-Cryst. Solids 3573261

Buraidah M H, Teo L P, Majid S R and Arof A K 2009 Physica B 4041373

Daniel M F, Desbat B, Cruege F, Trinquit O and Lassegues J C 1988 Solid State Ionics 28-30 637

Druger S D, Nitzam A and Ratner M A 1983 J. Chem. Phys. 79 3133

El Shafee E 1996 Carbohyd. Polym. 3193

Gogulamurali N, Suthanthiraraj S A and Maruthamuthu P 1992 Solid state ionics: Materials and applications (Singapore: World Scientific) p. 373

Gray F M 1991 Solid polymer electrolytes-Fundamental and technological applications (USA: VCH Publishers)

Guo J-H, Skinner G W, Harcum W W and Barnum P E 1998 Pharm. Sci. Tech. To. 1254

Hashmi S A, Kumar A, Maurya K K and Chandra S 1990 J. Phys. D23 1307

Hashmi S A, Chandra A and Chandra S 1992 in Solid state ionics: Materials and applications (eds) B V R Chowdari et al (Singapore: World Scientific) p. 567

Hema M, Selvasekerapandian S, Sakunthala A, Arunkuma D and Nithya H 2008 Physica B $\mathbf{4 0 3} 2740$

Idris N K, Nik Aziz N A, Zambri M S M, Zakaria N A and Isa M I N 2009 Ionics 15643

Jaipal Reddy M and Chu P P 2002 Electrochim. Acta 471189

Kadir M F Z, Aspanut Z, Majid S R and Arof A K 2011 Spectrochim. Acta A78 1068

Karan N K, Pradhan D K, Thomas R, Natesan B and Katiyar R S 2008 Solid State Ionics 179689

Kim C, Lee G, Liou K, Ryu K S, Kang S G and Chang S H 1999 Solid State Ionics 123251

Lopes L V S, Dragunski D C, Pawlicka A and Donoso J P 2003 Electrochim. Acta $\mathbf{4 8} 2021$

Ma X, Yu J, He K and Wang N 2007 Macromol. Mater. Eng. 292 503

Majid S R and Arof A K 2005 Physica B 35578

Majid S R and Arof A K 2007 Physica B 390209

Majid S R, Idris N H, Hassan M F, Winie T, Khiar A S A and Arof A K 2005 Ionics $\mathbf{1 1} 451$

Maurya K K, Srivastava N, Hashmi S A and Chandra S 1992 J. Mater. Sci. 276357

Michael M S, Jacob M M E, Prabaharan S R S and Radhakrishna S 1997 Solid State Ionics 98167 
Migahed M D, Ishra M, Fahmy T and Barakat A 2004 J. Phys. Chem. Solids $\mathbf{6 5} 1121$

Mishra R, Baskaran N, Ramakrishna P A and Rao K J 1998 Solid State Ionics 112261

Miyamoto T and Shibayama K 1973 J. Appl. Phys. 445372

Muralidharan P, Venkateswardu M and Satyanarayana N 2005 J. Non-Cryst. Solids 351583

Nadeem M, Akhtar M J, Khan A Y, Shaheen R and Haque M N 2002 Chem. Phys. Lett. 366433

Pandey K, Dwivedi M M, Tripathi M, Singh M and Agrawal S L 2008 Ionics 14515

Pandey K, Dwivedi M M, Singh M and Agrawal S L 2010 J. Polym. Res. 17127

Pawlicka A, Dragunski D C and Guimaraes K V 2004 Mol. Cryst. Liq. Cryst. 416105

Prabakar K, Narayandass S K and Mangalaraj D 2003 Mater. Chem. Phys. 78809

Pradhan D K, Coudhary R N P and Samantaray B K 2009 Mater. Chem. Phys. 115557

Psarras G C 2006 Compos. A-Appl. Sci. 371545

Pushpamalar V, Langford S J, Ahmad M and Lim Y Y 2006 Carbohydr. Polym. 64312

Rajendran S and Uma T 2000 J. Power Sources 87218

Ramesh S and Arof A K 2000 Solid State Ionics 1361197

Ramesh S and Arof A K 2001 Mater. Sci. Eng. B85 11

Ramly K, Isa M I N and Khiar A S A 2011 Mater. Res. Innovat. 1582

Ramya C S, Selvasekarapandian S, Savitha T, Hirankumar G, Baskaran R and Angelo P C 2006 Eur. Polym. J. 422672

Ratner M A 1987 Polymer electrolyte reviews-I (eds) J R MacCallum and C A Vincent (UK: Elsevier Applied Science) p. 173
Razak N R A and Winnie T 2009 Proceedings of national workshop on functional material, Centre of Ions, University of Malaya p. 55

Samsudin A S and Isa M I N 2012a J. Appl. Sci. 12174

Samsudin A S and Isa M I N 2012b Int. J. Polym. Mater. 6130

Samsudin A S, Kuan E C H and Isa M I N 2011 Int. J. Polym. Anal. Chem. 16477

Santa-Comba A, Pereira A, Lemos R, Santos D, Amarante J and Pinto M 2001 J. Biomed. Mater. Res. 55396

Schantz S and Torell L M 1993 Solid State Ionics 6047

Sekhon S S, Pradeep K V and Agnihotri S A 1998 Solid state ionics (eds) B V R Chowdari et al p. 217

Shuhaimi N E A, Teo L P, Majid S R and Arof A K 2010 Synthetic Met. 1601040

Singh P K, Bhattacharya B, Nagarale R K, Kim K W and Rhee H W 2010 Synth. Met. 160139

Song J Y, Wang Y Y and Wan C C J 2000 Electrochem. Soc. 147 3219

Souquet J L, Levy M and Duclot M 1994 Solid State Ionics 70 337

Sreepathi Rao S, Jaipal Reddy M, Laxmi Narsaiah E and Subba Rao U V 1995 Mater. Sci. Eng. B33 173

Stainer M, Hardy L C, Whitmore D H and Shriver D F 1984 J. Electrochem. Soc. 131784

Teeters D, Neuman R G and Tate B D 1996 Solid State Ionics 85 239

Wan Y, Peppley B, Creber K A M, Bui V T and Halliop E 2006 J. Power Sources 162105

Wang H, Tong Lin and Akif Kaynak 2008 Synthetic Met. 151136

Woo H J, Majid S R and Arof A K 2011 Solid State Ionics 19914

Wright P V 1975 Br. Polym. J. 7319 